\title{
Nouvelles mobilités pastorales : cas des éleveurs d'ovins de la wilaya de Djelfa, Algérie
}

\author{
Dihia Gaci ${ }^{1 *}$ Johann Huguenin ${ }^{2}$ Mohamed Kanoun ${ }^{3}$ \\ Jean-Pierre Boutonnet ${ }^{4}$ Hacène Abdelkrim ${ }^{1}$
}

\section{Mots-clés}

Ovin, transhumance, steppe, système pastoral, Algérie

Submitted: 11 May 2020

Accepted: 15 September 2020

Published: 1 March 2021

DOI: $10.19182 /$ remvt.36324

\section{Résumé}

Les évolutions socioéconomique, démographique, politique et climatique que la steppe algérienne a connues au cours des dernières décennies ont induit de profondes transformations au niveau des pratiques d'élevage, tout particulièrement en matière de mobilités pastorales. Cette étude décrit de nouvelles pratiques de mobilité adoptées actuellement par des éleveurs de la région de Djelfa. Elle s'est basée sur 59 entretiens semi-directifs réalisés entre 2014 et 2016 auprès d'éleveurs dans différentes zones d'accueil. Nos résultats ont mis en évidence une diversité de pratiques pastorales relative à la distance parcourue durant un cycle annuel, au calendrier, à l'itinéraire de transhumance et au passage ou non par le terroir d'attache. Ces mobilités pouvaient varier d'une année à l'autre ou au cours de la vie d'un éleveur. Cette étude a montré que de nouvelles formes d'adaptation au contexte changeant de la steppe algérienne pouvaient être mises en œuvre par les éleveurs par le moyen de nouvelles mobilités de grande distance. Nos résultats soulignent le renouvellement des formes de mobilité pastorale malgré le resserrement de l'espace et montrent que la sédentarisation n'est pas l'unique forme d'adaptation des populations pastorales aux changements.

- Comment citer cet article : Gaci D., Huguenin J., Kanoun M., Boutonnet J.-P., Abdelkrim H., 2021. New pastoral movements: the case of sheep herders in Djelfa Wilaya, Algeria. Rev. Elev. Med. Vet. Pays Trop., 74 (1): 3-11, doi: 10.19182/remvt.36324

\section{INTRODUCTION}

La mobilité a de tout temps constitué un important pilier dans la capacité d'adaptation des sociétés pastorales méditerranéennes face aux contraintes de leurs milieux (Nori, 2017). La littérature consacrée au pastoralisme ovin de la steppe algérienne décrit classiquement deux grands déplacements pendulaires : la Achaba qui est le déplacement estival vers les régions telliennes au nord de la steppe, et la Azaba, qui est le déplacement hivernal des troupeaux vers les régions présahariennes pour la

1. Ecole nationale supérieure agronomique d'El Harrach, avenue Hassan Badi, El Harrach, Alger, Algérie.

2. CIRAD, UMR SELMET, F-34398 Montpellier, France.

SELMET, Univ Montpellier, CIRAD, INRAE, Institut Agro, Montpellier, France.

3. Institut national de recherche agronomique Algérie, Djelfa.

4. INRA, UMR SELMET, F-34060 Montpellier, France.

SELMET, CIRAD, INRA, Montpellier SupAgro, Montpellier, France.

* Auteur pour la correspondance

Email : dihiag@yahoo.fr recherche de températures plus clémentes et de pâturages (Boukhobza, 1982 ; Chellig, 1992 ; Bourbouze, 2006 ; Nedjraoui et Bédrani, 2008). Ces mobilités ont pour but d'assurer, au long des saisons, des ressources pâturées pour le cheptel (Rondia, 2006 ; Bencherif, 2011).

Au cours des dernières décennies, la steppe algérienne a connu une évolution profonde. Sa population humaine a triplé en passant de 4 millions d'habitants en 1977 à 12 millions en 2010 (ONS, 2011), augmentant la pression des usages agricoles (élevage et culture) et non agricoles sur les terres de parcours. Par ailleurs, les effectifs d'animaux présents sur ces parcours sont eux aussi passés de 6 millions en 1968 à 18 millions en 2009 (Yabrir et al., 2015) ce qui aurait contribué à la régression de la disponibilité fourragère spontanée dans la steppe (Le Houérou, 1995 ; Daoudi et al., 2013 ; Hammouda et al., 2013). Les réformes foncières successives ont permis une expansion importante des cultures, des villes et des bourgs au détriment des pâturages et des couloirs de transhumance. L'usage massif d'apport de concentré, notamment de l'orge, dans la ration alimentaire des animaux, ainsi que l'association de l'agriculture au pastoralisme (pâturage de chaume, d'orge sinistrée, de jachère, entre autres) a permis à certains éleveurs de garantir l'alimentation de leurs animaux sans 
effectuer régulièrement de grands déplacements, pour se contenter de pâturages collectifs et privatifs disponibles dans leur wilaya d'origine (Aïdoud et al. 2006 ; Ghozlane et al., 2009). Cet ensemble de changements que la steppe a connus ces dernières décennies a fortement réduit les grands déplacements de troupeaux (Abbad et Genin, 2004)

Toutefois, selon Kanoun (2016), les grandes mobilités seraient toujours d'actualité dans les systèmes d'élevage pastoraux et agropastoraux des régions steppiques algériennes comme l'une des voies possibles d'adaptation de ces systèmes aux changements. La mobilité de longue distance représenterait une alternative à l'usage croissant de fourrages cultivés, mise en œuvre par une partie des éleveurs, variable selon les wilayas : le pourcentage d'éleveurs transhumants serait de $30 \%$ à Laghouat alors qu'il serait de $70 \%$ à Djelfa.

Comme l'indique Ben Hounet (2009) « [s'il] existe encore des « irréductibles » à la sédentarisation définitive », ces mobilités restent cependant peu étudiées. Elles sont le plus souvent décrites dans la littérature comme ayant fortement régressé suite à la sédentarisation accrue des éleveurs (Bourbouze et al., 2009) et classées selon la typologie, transhumant, semi-transhumant, et sédentaire (Bensouiah, 2005), avec peu d'informations.

La présente étude visait à caractériser, à travers le cas des éleveurs de Djelfa, les formes actuelles de mobilité des éleveurs de la steppe algérienne. Elle s'est appuyée sur l'analyse de Idda et al. (2017) qui montrent que des pratiques ancestrales peuvent perdurer sous des formes innovantes qui les confortent. Elle a tenté d'explorer l'hypothèse selon laquelle les systèmes pastoraux se caractériseraient par leur « aptitude à s'ajuster à des contextes géopolitiques, économiques et sociaux fluctuants et difficiles » (Ben Hounet, 2009). Il s'agissait ici de faire ressortir la diversité des pratiques de mobilités de longue distance de manière plus précise en vue d'en comprendre l'organisation, le fonctionnement et les facteurs qui influençaient les décisions des éleveurs.

\section{MATERIEL ET METHODES}

\section{Zone d'étude}

La steppe algérienne avec ses 20 millions de kilomètres carrés se trouve entre l'Atlas tellien et l'Atlas saharien en Algérie. La région de Djelfa, où a été effectuée cette étude, se trouve au cœur de la steppe et constituerait une zone de transit entre les hautes plaines du sud de l'Atlas tellien et les parcours sahariens. Sa superficie est de 33236 kilomètres carrés avec environ $85 \%$ de parcours steppiques. Cette région est aussi connue pour être la première productrice de viande ovine en Algérie avec un cheptel qui dépasserait 3,5 millions d'ovins, soit $14 \%$ du cheptel national, faisant de cette wilaya la première en termes d'effectifs ovins dans le pays (Kanoun, 2007). Elle possède le plus gros marché à bestiaux où se rencontrent les éleveurs et les demandeurs (Atchemdi, 2008). L'activité d'élevage constitue la principale source de revenus de la population locale. Le système d'élevage pratiqué dans ces territoires s'appuie sur la mobilité pastorale pour $72 \%$ des éleveurs (Kanoun, 2016).

Au cours des quatre dernières décennies, cette région a connu de profonds changements démographiques et dans le mode de vie de la population locale. Cette population est passée de 437000 habitants en 1987 à 1491370 habitants en 2013 (Kanoun, 2016). En 2013, 81 \% de cette population résidait dans des habitations en dur dans les agglomérations chefs-lieux de wilaya, alors qu'autrefois le mode de vie des éleveurs était majoritairement nomade (sous la tente). Les parcours ont eux aussi connu de fortes pressions sous l'effet de l'augmentation des effectifs d'ovins qui sont passés de 1 million en 1978 à 2 millions en 1997 et qui s'approcheraient actuellement de 4 millions (Bousaïd et al., 2018). La combinaison de ces facteurs a conduit à une forte concurrence sur l'espace steppique.

Située entre les isohyètes 100 et $300 \mathrm{~mm}$ (figure 1), la région étudiée connaît un climat méditerranéen à bioclimat aride, semi-aride à présaharien caractérisé par de fortes variabilités pluviométriques, avec une alternance entre périodes sèches et humides. Les périodes sèches les plus importantes ont été celles de 1940-1950, 1980-1985 et 19992004 (Belala et al., 2018).

Le paysage végétal de la steppe était autrefois dominé par des formations à alfa (Stipa tenacissima), sparte (Lygeum spartum) et armoise blanche (Artemisia herba-alba). Actuellement, on retrouve d'autres unités végétales représentant la dynamique régressive que connaît la steppe. Il s'agit d'unités principalement dominées par Atractylis serratuloides, Peganum harmala et Salsola vermiculata (Roselt, 2005 ; Hourizi et al., 2017).

Du fait des mutations rapides et profondes qu'elle a connues durant les dernières décennies, la région offre un terrain de choix pour étudier les

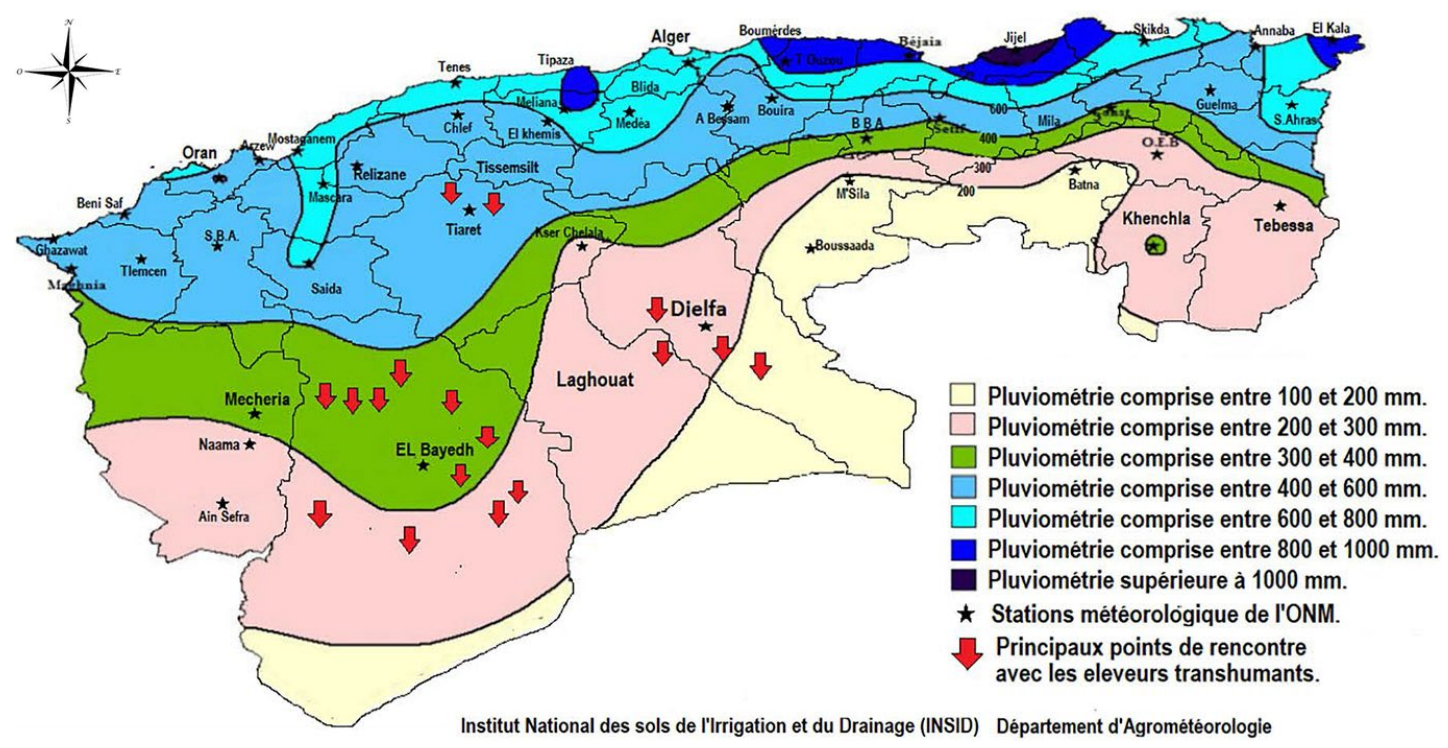

Figure 1 : Répartition des précipitations au nord de l'Algérie et principaux points de rencontre avec les transhumants. INSID, 2013, Carte d'occupation du sol, Bneder /// Rainfall distribution in Northern Algeria and main meeting points with transhumants. INSID, 2013, Land-use map, Bneder 
nouvelles formes de mobilités entreprises par les éleveurs pour faire face au contexte d'incertitudes variées que connaît la steppe algérienne. Les mobilités « de longue distance » prises en compte dans cette étude sont celles qui se pratiquent en dehors de la wilaya de Djelfa.

\section{Démarche méthodologique}

Pour étudier les mobilités pastorales de grandes distances, deux séries d'enquêtes ont été réalisées entre octobre 2014 et novembre 2016. Dans un premier temps (octobre 2014) une vingtaine d'entretiens exploratoires ont été menés auprès de personnes ressources (éleveurs, techniciens, institutionnels, ingénieurs, entre autres). Cette phase a été réalisée avec la collaboration des agents de l'Institut national de la recherche agronomique d'Algérie (INRAA) à Djelfa. Durant cette phase les éleveurs ont été rencontrés sur des parcours collectifs ou chez eux. Les résultats ont permis d'orienter les entretiens en mettant en évidence les principaux terroirs d'accueil des éleveurs qui pratiquaient les grandes mobilités, la saisonnalité et les raisons de leurs déplacements.

Dans un second temps (de février 2015 à novembre 2016) des entretiens plus approfondis avec les éleveurs ont été réalisés pour réunir les éléments permettant de comprendre les pratiques de mobilité et leurs motivations. L'échantillonnage des éleveurs s'est fait selon une combinaison de trois méthodes (Kherri, 2013 ; Kheffache, 2018) : a) l'échantillonnage « raisonné de convenance » réalisé dans le cas où les unités d'échantillonnage étaient faciles à joindre et disponibles pour répondre aux entretiens ; b) l'échantillonnage « selon le jugement» réalisé lorsqu'un éleveur ou un groupe d'éleveurs étaient jugés intéressants pour satisfaire les objectifs de cette étude ; des éleveurs principalement transhumants ont ainsi été sélectionnés ; et c) la méthode « boule de neige » lorsqu'un répondant indiquait un ou plusieurs éleveurs qui comportaient les caractéristiques recherchées, ici la mobilité de grande distance, définie comme la mobilité hors de Djelfa.

Au total, 59 éleveurs de la région de Djelfa pratiquant différents types de mobilité de grande distance ont été interviewés sur leurs terroirs d'accueil (zones de Laghouat, El Bayadh, Tiaret) ou sur leur terroir d'attache dans la wilaya de Djelfa (figure 1). Les informations ont été recueillies sur leurs déplacements sur une période de 10 ans en moyenne. Le critère « distance maximale parcourue durant un cycle annuel » a été retenu afin de classer les éleveurs. Ces entretiens ont été réalisés grâce à la collaboration des agents du Haut Commissariat du Développement de la Steppe (HCDS) dans les wilayas de Djelfa et d'El Bayadh, et des agents de la Conservation des Forêts (relevant de la Daïra d'Aflou et de Charef) ainsi que de quelques éleveurs. Les éleveurs ont été rencontrés sur leur lieu de pâturage : au niveau de parcours mis en défens par le HCDS, sur des terrains privés ou sur des parcours collectifs. Les campements et les habitations ont été repérés au fur et à mesure des déplacements ou grâce aux informations détenues par les agents du HCDS, de l'INRAA ou des forestiers.

Dans certains pays sahéliens comme au Niger ou au Sénégal (Hammel, 2001 ; Comité national du Code rural, Niger, 2010), « Le terroir d'attache est défini juridiquement comme étant une unité territoriale, déterminée et reconnue par les coutumes ou les textes de loi, à l'intérieur de laquelle vivent habituellement pendant la majeure partie de l'année des pasteurs et à laquelle ils restent attachés administrativement et culturellement lorsqu'ils se déplacent, que ce soit à l'occasion de la transhumance, du nomadisme ou des migrations. Ceci permet d'attribuer un droit d'usage prioritaire sur les ressources naturelles à la communauté de pasteurs attachée à ce terroir. » En Algérie, il n'existe pas de définition juridique officielle de «terroir d'attache » mais les éleveurs transhumants sont, comme les autres agriculteurs, enregistrés comme tels dans leur wilaya d'origine, même si leur troupeau paît hors de la wilaya la plupart du temps. Ce concept sera donc utilisé pour identifier le lieu d'appartenance des éleveurs transhumants de la wilaya de Djelfa.
Le guide d'entretien de la seconde phase comportait six volets : a) la famille et l'histoire de vie des éleveurs ; b) la structure de l'exploitation comme la taille du troupeau, les moyens détenus, le type d'habitat ; c) les mobilités pastorales, soit le type de déplacement, les raisons des déplacements, le choix des terroirs d'accueil, le calendrier des transhumances sur le court, moyen et long terme ; d) l'organisation des déplacements (aspects logistiques et relationnels), comportant entre autres les moyens de transport utilisés, le choix de la main-d'œuvre sollicitée, l'organisation familiale autour de cette pratique, le repérage des sites de transhumance, la location des parcours, le déplacement des troupeaux, des campements ; e) les relations sociales impliquées, comme le/la propriétaire des terres et des camions loués, la garde des enfants, les liens sociaux avec le berger, l'importance de la dimension sociale dans la pratique de transhumance ; et f) les ressources utilisées pour la conduite du troupeau, notamment l'accès à l'eau, le choix du type de parcours, les types de végétation qui les caractérisent, la quantité de concentré utilisé selon la période de l'année.

\section{Traitement des données}

Une préanalyse du contenu des enquêtes a été réalisée pour faire ressortir les variables les plus importantes. Celles-ci ont été codifiées, catégorisées et classées dans un tableau Excel qui comprenait 90 variables. Pour l'étude sur les mobilités de déplacement sur longues distances, 15 variables pertinentes ont été sélectionnées : passage ou non par le terroir d'attache durant l'année, diversité des lieux de passage pour chaque saison, taille du troupeau, possession de terres personnelles ou non, distance minimale et maximale parcourue pour chaque saison, distance maximale et minimale parcourue durant l'année, distance moyenne parcourue pour chaque éleveur. Parmi les 15 variables, sept étaient qualitatives. Les données relatives à ces dernières ont été transformées en modalités ou codes. Le total de ces modalités est de 21 (tableau I). Les informations de la base de données ont été recoupées afin de mettre en évidence les typologies de mobilités existantes selon la proximité des pratiques des différentes exploitations.

Pour cela des opérations statistiques comme des corrélations, des comptages, des pourcentages ou des descriptions ont été réalisés (Wenlin, 2007). Ceci a permis de décrire la diversité des mobilités pastorales rencontrées, leurs singularités, mais aussi leur originalité par rapport à ce qui existe dans la littérature. La diversité des grandes mobilités pastorales rencontrées est présentée dans la figure 2. Notre étude rend compte des informations recueillies concernant les déplacements des éleveurs durant la dernière décennie écoulée. Ces informations ont permis de caractériser trois types de déplacement de longue distance (cf. plus loin). L'appartenance à l'un de ces trois types n'est pas définitive : au cours de leur vie ou de la vie de leur famille, les itinéraires et les distances peuvent évoluer. Certains éleveurs qui transhumaient loin de leur wilaya se sont repliés sur leur terroir d'origine, d'autres ont inauguré des transhumances de grande amplitude (Gaci et Boutonnet, 2020).

\section{RESULTATS}

Les principales caractéristiques discriminantes des mobilités des éleveurs ont été a) la distance parcourue, b) l'itinéraire, et c) le passage ou non par le terroir d'attache.

\section{Transhumance à distances diverses}

Dans l'échantillon, trois éleveurs ont parcouru des distances qui pouvaient atteindre $3000 \mathrm{~km}$ durant un cycle annuel. Au cours de ce cycle, la majorité, soit 34 sur 59 éleveurs, transhumait sur de longues distances qui avoisinaient $1500 \mathrm{~km}, 15$ éleveurs parcouraient environ $800 \mathrm{~km}$ en moyenne, seuls 7 éleveurs se déplaçaient sur des distances de moins de $500 \mathrm{~km}$ (figure 2). Des éleveurs de troupeaux de tailles très diverses pouvaient avoir les mêmes comportements de 
seulement le déplacement des animaux vers les zones d'accueil mais aussi la surveillance des animaux au pâturage, l'approvisionnement en eau, l'installation des campements, les relations avec les populations locales et les services administratifs, etc. Nos enquêtes ont révélé que pour ceux qui ne disposaient pas des relations sociales variées nécessaires, l'accès aux ressources s'avérait difficile, non rentable et parfois impossible en raison de la grande pression sur les pâturages. La disponibilité de ce « capital social » pouvait se retrouver chez des éleveurs possédant des troupeaux de toute taille, de même de grands éleveurs pouvaient ne pas disposer des ressources sociales nécessaires.

\section{Itinéraire et calendrier de déplacement}

Les itinéraires et les distances parcourues pour chaque éleveur ont permis d'identifier trois types de transhumance de longue distance au cours d'un cycle annuel (figure 4).

\section{Déplacement de moyenne amplitude}

Les figures 4 et 5 montrent que les éleveurs pratiquant des déplacements de moyenne amplitude, environ 500-800 kilomètres, transhumaient vers les régions présahariennes des wilayas limitrophes de Laghouat et d'El Bayadh. Ils allaient vers ces régions en hiver afin de retrouver des températures plus clémentes, de l'espace, de la sécurité et un peu de plantes d'abroutissement. Ils ne cherchaient pas nécessairement un parcours pour la qualité de sa végétation, raison pour laquelle ils complémentaient leur troupeau avec des concentrés (surtout de l'orge). Leur but était d'assurer pour les brebis des mises bas dans de bonnes conditions et de minimiser les pertes d'agneaux dues au froid ou au vol. Au début du printemps, les éleveurs revenaient vers les zones steppiques d'altitude (800-1200 m) et y louaient des terres mises en défens ou des jachères. En été ces éleveurs louaient des chaumes ou des céréales sinistrées au nord en région tellienne et plus

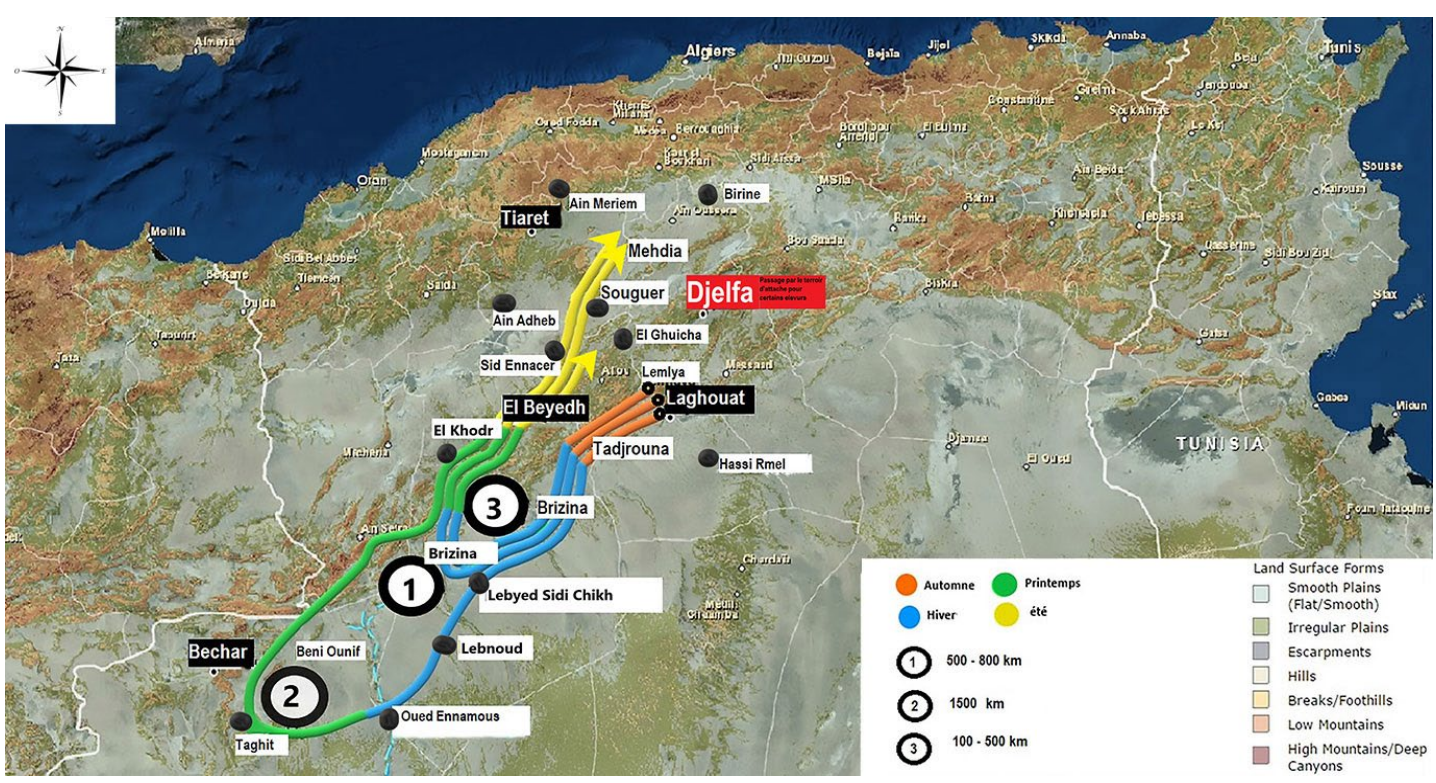

Figure 4 : Principaux itinéraires de transhumance au nord de l'Algérie /// Main transhumance routes in Northern Algeria
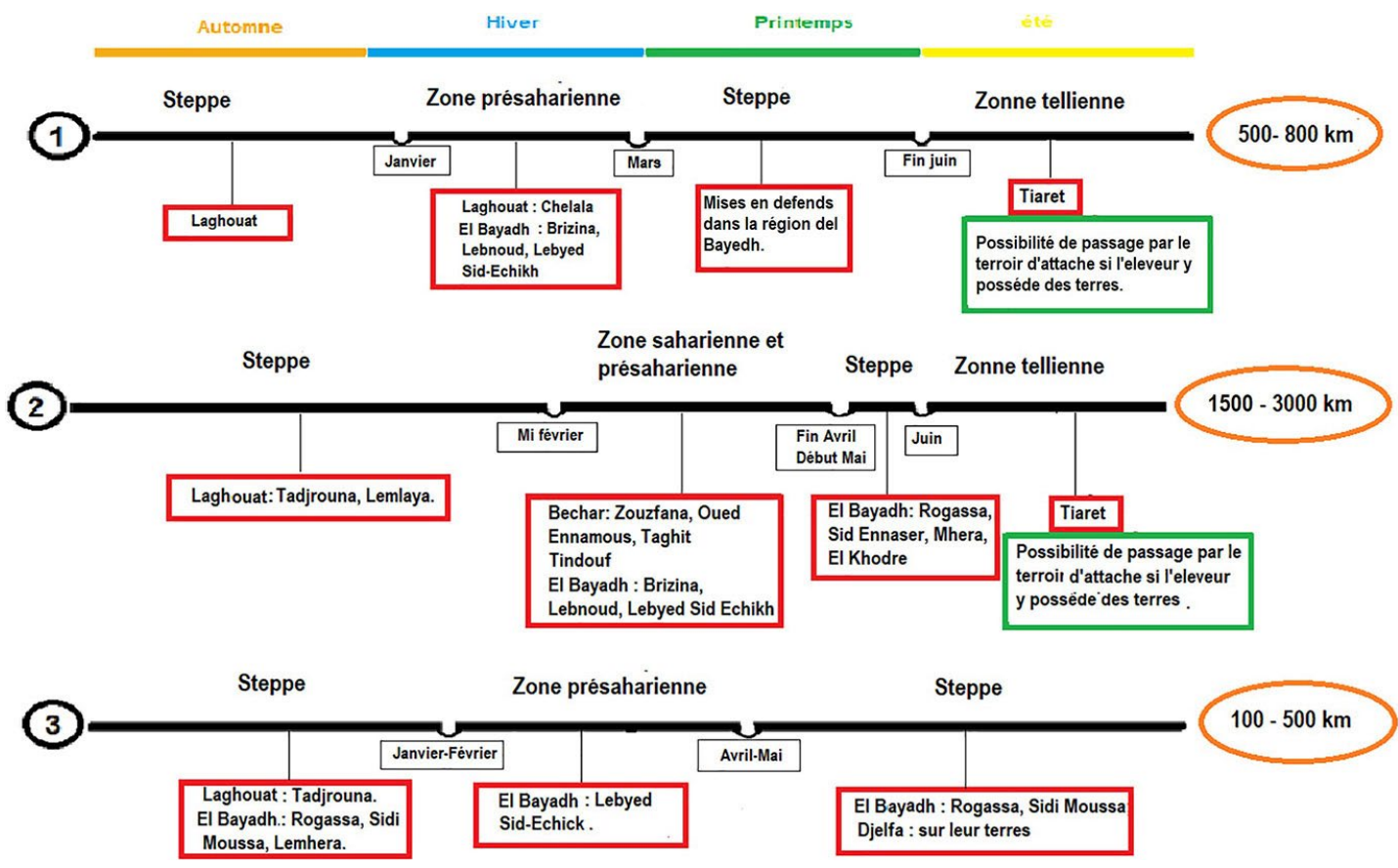

Figure 5 : Calendrier de transhumances au nord de l'Algérie /// Calendar of transhumance in Northern Algeria 
fréquemment dans la région de Tiaret. Cette région est très appréciée pour son espace et la qualité de ses terrains. Dans le cas où un éleveur avait des terres dans son terroir d'attache, il pouvait y passer l'été ou y faire seulement un passage pour profiter des chaumes disponibles même en faible quantité. Les surfaces louées étaient en fonction de la taille du troupeau et pouvaient aller jusqu'à 100 hectares. Les éleveurs effectuant cet itinéraire possédaient des troupeaux de taille moyenne de 200 à 600 brebis.

Le choix de cet itinéraire ne semblait pas être lié exclusivement aux moyens économiques des éleveurs. Les éleveurs de ce groupe auraient choisi des itinéraires rentables avec un minimum de contraintes en termes de transport et de difficultés d'accès au terrain. Certains d'entre eux ont expliqué lors de l'entretien qu'il était plus contraignant pour eux de s'aventurer vers des zones comme Bechar. Cela leur demandait une organisation importante et parfois peu rentable. Des liens sociaux leur permettaient de garder une certaine stabilité dans la pratique de ce type de mobilité. En effet, ils louaient des chaumes et des jachères chez les mêmes agriculteurs depuis un certain nombre d'années, ce qui semblait être un acquis précieux que les éleveurs veillaient à préserver. D'autres éleveurs préféraient, durant la période estivale, rester dans leur terroir d'attache. Ils faisaient alors pâturer leurs troupeaux sur des parcours collectifs pauvres en ressources fourragères et devaient acheter de l'orge pour assurer la couverture des besoins des animaux.

\section{Déplacement de grande amplitude}

Le déplacement de grande amplitude se caractérisait par un long passage dans la steppe, du début de l'automne jusqu'au milieu de l'hiver. Durant cette période, les éleveurs demeuraient au pied des zones montagneuses des steppes situées dans la wilaya de Laghouat ou d'El Bayadh afin de s'abriter des vents froids. Quand l'année était annoncée favorable (sur le plan pluviométrique et donc en matière de végétation pastorale) dans les régions sahariennes, les éleveurs s'y rendaient et y restaient jusqu'au milieu du printemps. Cet itinéraire vers les régions sahariennes était emprunté quand les éleveurs estimaient de visu ou par leurs informateurs de confiance que la végétation éphémère, appelée $a c h e b s$, poussait bien après les pluies pour assurer une ressource fourragère gratuite pendant plusieurs mois (Chehmaet al., 2005). Si ce n'était pas le cas, les éleveurs préféraient rester dans la steppe pour louer des terres mises en défens auprès du HCDS ou des jachères en zones telliennes auprès d'agriculteurs. Les déplacements de fin d'hiver vers le Sahara se faisaient généralement en camion en direction de régions comme Tindouf, Bechar. Ils pouvaient même arriver jusqu'aux frontières libyenne ou marocaine, où se tenaient des marchés interrégionaux importants. Cela permettait d'organiser plus efficacement et plus rapidement la vente d'animaux, les éleveurs disposant ainsi de liquidités financières pour acheter des aliments pour bétail et pallier le manque de fourrage sur les parcours (Alary et Boutonnet, 2006).

Les éleveurs qui faisaient ce type de transhumance possédaient généralement un camion. Pour transporter la totalité de leurs animaux, ils faisaient appel à d'autres éleveurs de confiance, possédant un camion, préférablement de la même tribu, afin qu'il n'y ait pas de vol en cours de route. Ces opérations n'étaient accessibles qu'aux éleveurs disposant de bonnes relations sociales dans leur wilaya et dans les wilayas d'accueil. Le coût de la location était de 40 DA/ $/ \mathrm{km} /$ camion (30 centimes d'euros) sur les routes goudronnées et de $50 \mathrm{DA} / \mathrm{km} / \mathrm{camion}$ (35 centimes) pour le transport en piste.

Selon les éleveurs, les parcours sahariens, quand ils étaient verdoyants, permettaient aux animaux de se nourrir exclusivement d'herbe durant près deux mois sans apport de compléments. Cela permettait aux éleveurs de réaliser des économies. En parallèle, les éleveurs réalisaient de grandes ventes d'animaux au niveau des marchés de ces régions proches des frontières.
Au milieu du printemps, quand la chaleur en région saharienne devenait élevée pour les animaux, le retour vers les zones steppiques se faisait généralement à pied sur plusieurs jours (15 à $20 \mathrm{~km} / \mathrm{jour}$ ) pour permettre aux brebis de brouter la végétation au cours de leur déplacement. Les éleveurs faisaient ainsi des économies sur le transport des animaux. Lors de ce déplacement, le campement était transporté par un camion qui appartenait à l'éleveur.

Dans la steppe les éleveurs faisaient escale en louant des mises en défens au HCDS ou en occupant des terres collectives en attendant de pouvoir se déplacer vers le nord, dans les régions telliennes, sur les chaumes ou les céréales sinistrées qu'ils avaient réservés à des agriculteurs chez lesquels ils avaient l'habitude de séjourner. La relation de confiance qui s'était établie entre l'éleveur et l'agriculteur était un des critères de choix des parcelles louées par l'éleveur. Parfois le choix des surfaces louées était défini selon le tarif proposé par l'agriculteur, la qualité des chaumes ou des céréales sinistrées. La situation de la parcelle par rapport aux points d'eau, aux marchés locaux ou à la ville semblait définir les critères de choix de l'éleveur. Il est apparu au cours des enquêtes que les éleveurs les moins avantagés en termes de moyens financiers n'avaient pas accès aux meilleures parcelles. Il leur arrivait de ne pas pouvoir louer certaines années car les prix étaient soit trop élevés pour eux, soit il n'y avait plus de parcelles disponibles. Si les éleveurs possédaient des terres dans leurs terroirs d'attache ils pouvaient y faire un passage en été lorsqu'ils jugeaient que cela en valait la peine. En effet si l'année n'avait pas été favorable et les chaumes étaient peu abondants, les éleveurs n'y passaient pas. Les distances parcourues dans l'année par ces éleveurs pouvaient dépasser 1500 kilomètres.

La grande contrainte rencontrée dans ces zones était le manque d'eau. Les éleveurs parcouraient jusqu'à 150 kilomètres depuis leur campement pour en chercher soit chez des particuliers soit à partir de fontaines ou de puits publics, à une fréquence d'une fois tous les deux ou trois jours selon les besoins en eau. Le transport de l'eau se faisait dans des barils ou des citernes transportées en camion. Un seul éleveur de l'échantillon, possédant 2000 animaux, disposait d'un camionciterne. Lors de ces déplacements, les éleveurs devaient disposer de main-d'œuvre familiale ou salariée pour effectuer différentes tâches comme le gardiennage, le transport de l'eau et de nourriture. Afin d'assurer leur sécurité dans ces endroits très isolés où les risques de pannes ou de vols pouvaient être fatals, les éleveurs se déplaçaient en groupes de deux à dix campements appartenant aux membres de la même famille ou à leur tribu.

La pratique de ce type de transhumance requiert un réseau de connaissances sociales. Cela est nécessaire pour faciliter l'accès aux ressources pastorales (pâturages, eau) sur des zones éloignées et difficiles comme le Sahara. Des zones sont très convoitées comme les mises en défens, à El Bayadh par exemple, ou les chaumes et les céréales sinistrés dans les régions telliennes, par exemple à Tiaret. Ces relations se tissent au cours du temps, sur le moyen ou long terme, sur la base de liens marchands de confiance qui s'établissent lors de la location de terres, de ventes d'animaux ou d'accès à l'eau, ou encore lors des rencontres sur les lieux de transhumances ou sur les marchés. Ces contacts étaient soigneusement entretenus par les éleveurs dans le cas où il y aurait eu nécessité de demander des informations ou d'avoir recours à des échanges de services.

Cette catégorie d'éleveurs adaptait ses itinéraires de transhumance aux variations interannuelles de la pluviométrie ainsi qu'aux bonnes opportunités de pâturages qui s'offraient à eux. Ceux-ci pouvaient être différents d'une année à l'autre. Certaines années les distances parcourues dans un cycle annuel ne dépassaient pas 200 kilomètres. Finalement l'itinéraire et la distance effectués dépendaient d'un compromis toujours remis en question entre l'existence ou la qualité de la ressource (herbe, eau, conditions météorologiques) et son accessibilité (prix de la ressource et du moyen de transport, maîtrise des relations sociales). 


\section{Déplacement de petite amplitude}

Les éleveurs pratiquant une transhumance de petite amplitude se déplaçaient vers des zones peu éloignées de leur terroir d'origine et pour des durées courtes. Ils passaient l'hiver dans les régions présahariennes comme à Brizinaet Lebyed Sidi Echikh (El Bayadh) pour les raisons évoquées précédemment pour les éleveurs pratiquant les déplacements de moyenne amplitude. Au printemps ils revenaient dans la steppe pour louer des terres mises en défens par le HCDS. Les surfaces louées pouvaient varier d'un éleveur à l'autre selon la taille du troupeau et la disponibilité des terres. Le coût de la location pour environ deux mois était de $1000 \mathrm{DA} / \mathrm{ha}(7 € / \mathrm{ha})$, et de $2000 \mathrm{DA} / \mathrm{ha}(14 € /$ ha) pour les plantations en plantes fourragères comme Atriplex. L'argent était reversé dans la trésorerie de la commune. Cependant tous les éleveurs n'y avaient pas accès. De nombreux éleveurs ont confié avoir des difficultés à trouver des terres mises en défens à louer, surtout les petits éleveurs possédant moins de 150 têtes. Il était parfois nécessaire de s'y prendre plusieurs mois à l'avance pour réserver une place et très souvent le nombre d'hectares attribués aux éleveurs était insuffisant. Les charges pastorales autorisées dans la steppe n'étaient donc pas toujours respectées. Les demandes des éleveurs étant nombreuses et les cheptels importants, les parcours mis en défens se retrouvaient souvent surpâturés. Les responsables du HCDS ont confié avoir rencontré beaucoup de difficultés à réguler les charges animales en raison de l'importance des troupeaux et de la pression exercée par les éleveurs. D'autre part, les éleveurs disaient que l'offre fourragère des parcours mis en défens restait très faible, surtout les années où il ne pleuvait pas.

Les animaux se nourrissaient surtout de l'herbe qui poussait après les pluies. Les plantes ligneuses étaient utiles pour leurs apports en fibres dans le cas où les éleveurs étaient contraints d'acheter du grain à des prix de l'ordre de $3500 \mathrm{DA} / \mathrm{q}$ ( $25 € / \mathrm{q})$ pour compléter la ration alimentaire des animaux à raison de $1 \mathrm{~kg} /$ tête/jour en moyenne. Cette quantité pouvait augmenter jusqu'à $2 \mathrm{~kg}$ en cas de disette. Les terres mises en défens étaient louées en réalité afin d'assurer une tranquillité et une sécurité aux éleveurs et à leurs animaux. Selon eux, il était de plus en plus difficile de trouver des terres pour faire pâturer leurs animaux car l'appropriation des terres à des fins de constructions ou de mise en culture commençait à se généraliser.

En été, ces éleveurs restaient dans la steppe sur des terres collectives dont l'accès était contrôlé par des tribus du nord d'El Bayadhvers Rogassa. Ils pouvaient aussi retourner à Djelfa sur leurs propres terres ou sur les terres collectives de leur propre tribu. Cependant la végétation y est pauvre et ne couvre que les besoins en fibres. Les éleveurs qui empruntaient cet itinéraire préféraient nourrir leurs animaux au grain en été et les faire pâturer sur de la végétation maigre que de louer des chaumes. Le choix de cet itinéraire semblait être dû aux difficultés économiques et au manque de relations sociales pour trouver des terres de location et pour se déplacer vers les régions sahariennes. Les distances parcourues durant l'année par ces éleveurs ne dépassaient pas 500 kilomètres.

\section{Un retour au terroir d'attache souvent problématique}

Tous les éleveurs ne passaient pas par leur terroir d'attache avec leurs troupeaux, la région de Djelfa ayant connu une fermeture des espaces de plus en plus prononcée par l'urbanisation et la mise en culture des terres. Les éleveurs ne trouvaient plus où faire pâturer les animaux dans leur terroir d'attache. Il était presque nécessaire à chaque fois de les faire déplacer en camion d'un site à l'autre. Notons que certains éleveurs revenaient pour une période de l'année dans leur terroir d'attache. Parmi les 59 éleveurs interviewés, 30 ont déclaré y séjourner avec leurs troupeaux au moins à un moment de l'année. Les périodes de passage pouvaient varier de deux semaines à une saison entière voire deux. Les raisons évoquées étaient la disponibilité en herbe dans les parcours collectifs ou sur leurs propres terres, ou encore le déplacement pendant une année de sécheresse sur tout le territoire algérien. Sur les 30 éleveurs qui revenaient à Djelfa, 23 disposaient dans leur terroir d'attache de droits d'accès exclusifs aux parcours octroyés par les autorités locales. Le reste des éleveurs (29) ne revenaient quasiment pas dans leur terroir d'attache avec leurs troupeaux. Ils ont indiqué ne pas y trouver de place pour faire pâturer les animaux. Ils avaient bien conscience que les parcours de Djelfa étaient saturés. Ils ne songeaient même pas à y revenir un jour. Ils erraient entre la steppe des hautes plaines et les régions sahariennes à la recherche de pâturages et ne revenaient sur leur territoire que pour des nécessités administratives ou familiales.

\section{DISCUSSION}

Cette étude a décrit une réalité rarement documentée dans la littérature. Les informations recueillies ont montré la complexité des mobilités de longues distances pratiquées par les éleveurs de la région de Djelfa, qui résultait, d'une part, de l'étendue du territoire parcouru, d'autre part, du perpétuel mouvement des éleveurs dans ces zones. Les enquêtes ont mis en évidence l'existence toujours actuelle de mobilités de longue distance pratiquées de façon pérenne et selon des modalités variées de la part d'éleveurs de la wilaya de Djelfa. Ce constat remet en cause les propositions de Bensouiah (2005) et Bencherif (2011). Selon eux, l'abandon des grandes mobilités serait dû à la diminution des parcours, au rétrécissement des couloirs de transhumances et à l'adoption d'un mode de vie sédentaire par les éleveurs. La présente étude a permis d'établir, au contraire, que ces contraintes avaient incité certains éleveurs à innover en matière de mobilité pastorale afin de maintenir leur élevage a) dès les années 1970 grâce au transport par camion des animaux et de l'eau, et b) depuis les années 2000 grâce à la sécurité recouvrée dans les vastes territoires du Sahara et aux progrès des télécommunications qui ont favorisé la mobilisation de leurs réseaux sociaux. C'est ainsi que certains éleveurs parcourent de très longues distances et n'hésitent pas à explorer de nouvelles destinations afin de trouver des pâturages intéressants. Certains d'entre eux se déplacent toute l'année sans revenir à leur terroir d'attache. Selon Bourbouze et al. (2009) et Kanoun et al. (2018), l'accès à l'information via le téléphone portable et le développement des réseaux routier et hydraulique sur l'ensemble du territoire algérien, ainsi que l'acquisition de moyens motorisés modernes ont permis d'accéder plus facilement à de nouveaux espaces mais aussi aux marchés locaux ou interrégionaux.

Les déplacements vers les zones de transhumance se décident plus vite et se font sur de plus longues distances. Pour réduire leur vulnérabilité résultant de la faible productivité des parcours pastoraux, les éleveurs doivent envisager un minimum de mouvement afin d'assurer les besoins alimentaires de leur troupeau et de minimiser le recours aux sous-produits des cultures et aux concentrés. C'est ainsi que les éleveurs amènent leurs troupeaux et leurs citernes en camion vers des régions où l'accès à l'eau reste difficile. Ils vont vers les régions sahariennes et présahariennes dès qu'une précipitation engendre la germination des végétations éphémères. Cette pratique n'était pas envisagée chez les éleveurs traditionnels steppiques. Selon Kheldoun (2000) les éleveurs sans moyens ont tendance à se replier sur une partie de leurs terrains privés et à s'y fixer toute l'année. Selon Kanoun (2016), la sédentarisation reste une logique minoritaire dans la région de Djelfa dans le rapport à l'espace des éleveurs.

Outre les moyens matériels, l'accès aux pâturages par transhumance demande notamment des facultés d'organisation, de bonnes ressources relationnelles. Nos enquêtes confirment les résultats de Kanoun et al. (2013) selon lesquels les ressources relationnelles sont très importantes pour maintenir l'élevage dans ces milieux. Ce sont donc généralement des éleveurs disposant de moyens financiers et 
d'un capital social importants qui s'aventurent à ce genre de pratique. Selon les éleveurs interviewés, les parcours deviennent de plus en plus inaccessibles du fait de l'appropriation privative des terres collectives locales qui commence à se faire ressentir même dans les parcours présahariens et sahariens, accroissant la pression pour des déplacements sur des distances de plus en plus longues. La concurrence pour la location des parcours et des chaumes commence à devenir importante. Les agents du HCDS notent eux aussi une pression grandissante sur les parcours mis en défens. Selon eux, les espaces accordés aux mises en défens actuelles ne sont pas suffisants pour satisfaire la demande des éleveurs durant la saison printanière. A l'avenir, les parcours steppiques, présahariens et sahariens pourraient connaître une appropriation individuelle de plus en plus importante qui pourrait être marquée par le développement de la mise en culture des parcours et de l'urbanisation. Cette situation pourrait notamment s'accompagner d'un appauvrissement des parcours de plus en plus important. S'ajouterait à cela l'accroissement du cheptel et du nombre d'éleveurs dans ces territoires. Des arbitrages entre ces usages concurrents de l'espace pastoral (agriculture, urbanisation, pastoralisme) dépendront le développement des nouvelles pratiques de l'élevage pastoral algérien.

\section{- CONCLUSION}

Cette étude a mis en évidence de nouvelles organisations des grandes mobilités chez les éleveurs de la wilaya de Djelfa. Les différentes formes de transhumance identifiées se distinguent par leurs itinéraires, les distances parcourues au cours d'un cycle annuel et le calendrier de transhumance. Nous avons montré que les mobilités sont toujours d'actualité en territoire steppique et que les éleveurs s'adaptent à leur contexte en mobilisant des moyens matériels et humains pour y parvenir. La mobilité reste un pilier central dans les systèmes d'élevage des régions steppiques. Ces résultats réfutent l'hypothèse de sédentarisation irrémédiable des systèmes pastoraux. Ils remettent aussi en cause les analyses selon lesquelles les diminutions des superficies des parcours et des couloirs de transhumances entraîneraient un abandon progressif des pratiques de mobilité de grande distance (Abbad et Genin, 2004 ; Bensouiah, 2005 ; Aïdoud et al., 2006 ; Bencherif, 2011). De fait, les transhumances de longue distance, notamment celles de moyenne et grande amplitude, sont un des outils mis en œuvre par les éleveurs qui le peuvent pour pallier la réduction des ressources pastorales de la steppe. Ces déplacements peuvent s'effectuer sur toute l'année sans passer par le terroir d'attache, déjà saturé. Actuellement les mobilités de grande amplitude sont l'un des outils utilisés par les éleveurs de Djelfa pour maintenir leurs systèmes d'élevage. Les moyens motorisés, les technologies comme les téléphones portables, le développement du réseau routier, des forages d'eau à travers le territoire national, la sécurité recouvrée ainsi que le prix abordable du carburant ont grandement facilité l'accès à cette pratique. Cependant l'accès aux ressources reste problématique : il nécessite la mobilisation de capitaux relationnels et financiers importants. Quand les éleveurs n'ont pas accès à ces ressources, n'étant pas inséré dans les «bons » réseaux sociaux et ne disposant pas d'avance de trésorerie substantielle, ils sont obligés, en restant sur leur terroir d'attache ou dans ses environs immédiats, de recourir à des achats d'aliment grossier et concentré, ce qui peut menacer la rentabilité de leur production. Finalement, la mobilité de longue distance, l'achat d'aliment pour bétail, le pâturage sur des parcours préservés sur le terroir d'attache sont autant de ressources complémentaires utilisées pour s'adapter aux aléas climatiques, sociaux ou économiques, par les éleveurs d'ovins de Djelfa selon leurs besoins et les opportunités qu'ils ont de les mobiliser.

\section{Remerciements}

Nous remercions la direction de l'UMR SELMET à Montpellier pour son accueil et son appui logistique et méthodologique. Nous remercions vivement les cadres, ingénieurs et techniciens du HCDS de Djelfa et El Bayadh, et particulièrement Monsieur le Commissaire général Amedjkouh Moustafa et les ingénieurs Makhlouf Aissa du HCDS de Djelfa, le commissaire de la Wilaya d'El Bayadh Ahmed Moussa et toute son équipe, en particulier Laarbi Moulay, pour leur disponibilité, leur accueil et leur grand appui sur le terrain. Nous remercions notamment l'ensemble des cadres et des techniciens de l'INRAA Djelfa qui ont participé à la direction des enquêtes. Nous remercions la Conservation des Forêts d'Aflou et de Djelfa pour leur appui sur le terrain. Nous remercions notamment la famille Amedjkouh pour son accueil durant la période d'enquête dans la région de Djelfa. Nos vifs remerciements vont à toute personne ayant contribué à la réalisation de cette étude, en particulier à Dellaoui Boualem, Bedak Menad, Loumasine Hiba, Gaci Idir, Gaci Kenza et Tahri Redouan. Nous remercions l'ensemble des éleveurs ayant participé à cette étude. Enfin nous remercions les relecteurs anonymes dont les conseils judicieux nous ont permis d'enrichir notre texte.

\section{Conflits d'intérêts}

Les auteurs déclarent que l'étude a été menée sans conflit d’intérêts.

\section{Déclaration des contributions des auteurs}

DG a participé à la conception et à la planification de l'étude, réalisé les enquêtes de terrain, collecté les données, construit leur analyse et leur interprétation, rédigé la première version et les versions successives du manuscrit; $\mathrm{JH}$ a contribué à poser la problématique et a participé à la rédaction de l'article ; MK a conçu le projet et organisé les enquêtes ; JPB a participé à l'interprétation des données d'enquête, à leur mise en forme et à la rédaction de l'article ; HA a supervisé l'ensemble du travail et participé à la conception de la recherche.

\section{REFERENCES}

Abbad A., Genin D., 2004. Politiques de développement agropastoral au Maghreb : enseignement pour de nouvelles problématiques de recherche développement. In: Environnement et société en mutation (Ed.Picouët M. et al.). IRD, Paris, France, 341-358, doi: 10.4000/books.irdeditions.1146

Aïdoud A., Le Houérou H.N., Le Floch E., 2006. Les steppes arides du Nord de l'Afrique. Sécheresse, 17 (1-2): 01-06

Alary V., Boutonnet J.P., 2006. L'élevage ovin dans l'économie des pays du Maghreb : un secteur en pleine évolution. Sécheresse, 17 (1-2): 40-46

Bencherif S., 2011. L'élevage pastoral et la céréaliculture dans la steppe algérienne : Évolution et possibilités de développement. Thèse Doct., Agroparistech, Paris, France, $268 p$.

Belala F., Hirche A., Muller S.D., Tourki M., Salamani M., Ait Hamouda T., Boughani M., 2018. Rainfall patterns of Algerian steppes and impacts on natural vegetation in $20^{\text {th }}$ century. J. Arid Land 10: 561-573, doi: 10.1007/ s40333-018-0095-x

Ben Hounet Y., 2009. Le poids du nomadisme pastoral dans les steppes algériennes. Études Rural. 184: 107-122, doi: 10.4000/etudesrurales.10514

Bensouiah R., 2005. Pasteurset agro-pasteurs de la steppe algérienne enquête sur la région de Djebel Amour. Revue Strates 11: 1-14, doi: 10.4000/strates.478

Boukhobza M., 1982. L'agro-pastoralisme traditionnel en Algérie : de l'ordre tribal au désordre colonial. Office des Presses Universitaires, Alger, Algérie, 458 p.

Bourbouze A., Ben Saad A., Chiche., Jaubert R., 2009. Sauvegarder des terrains collectifs et de parcours. In Hervieu B (dir.), Thibault H-L (dir.), Abis S (coord.), «Repenser le développement rural en Méditerranée», Mediterr, 11: $243-275$

Bourbouze A., 2006. Systèmes d'élevage et production animale dans les steppes du nord de l'Afrique: une relecture de la société pastorale du Maghreb. Sécheresse 17 (1-2): 31-39

Bousaïd A., Souhair N., Du Bois C., Schmitz S., 2018. L'amplification de la désertification par les pratiques agro-sylvo-pastorales dans les hautes plaines steppiques algériennes : les modes d'habiter de la Wilaya de Djelfa. Envi. Nat. Lands. 28 p. doi: 10.4000/cybergeo.29257 
Chehma A., Djebar M.R., Hadjaiji F., Rouabeh L., 2005. Etude floristique spatio-temporelle des parcours sahariens du Sud-Est algérien. Sécheresse $\mathbf{1 6}$ (4) : 275-85

Chellig R., 1992. Les races ovines algériennes. Office des Presses Universitaires, Alger, Algérie, $80 \mathrm{p}$.

Comité National du Code Rural (Niger), 2010. Article 2 de l'ordonnance n²010-029 du 20 mai 2010 relative au pastoralisme et article 2 du décret $n^{\circ}$ 97-007 du 10 janvier 1997 fixant le statut des terroirs d'attache des pasteurs, www.coderural-niger.net/spip.php?article295 (consulté le 15 novembre 2019)

Daoudi A., Terranti S., Hammouda R.F., Bedrani S., 2013. Adaptation à la sécheresse en steppe algérienne : le cas des stratégies productives des agropasteurs de Hadj Mechri. Cah. Agri. 22 (4): 303-310, doi: 10.1684/ agr.2013.0629

Gaci D., Boutonnet J.P., 2020. Novel alternative strategies for resilience towards climatic and socio-economic changes: an analysis of life trajectories of pastoralists in Algerian steppe. 7th International Symposium for Farming System Design, Tunis, Tunisie, 21-24 March 2021

Ghozlane F., Bendir M., Yakhlef H., Marie M., 2009. La sédentarisation et le développement durable de l'élevage ovin dans les zones steppiques algériennes. Cas de la wilaya de Djelfa. Rencontres Rech. Rumi., 16: 126

Hammel R., 2011. Terroirs d'attache des pasteurs au Niger. www. pubs.iied. org/pdfs/9069lIED.pdf (consulté 19 nov 2019)

Hammouda R.F., Huguenin J., Nedjraoui D., 2013. Régression des parcours et adaptation des élevages en zone steppique algérienne. Rencontres Rech. Rum. 20: 299

Hourizi R., Hirche A., Djellouli Y., 2017. Changements spatio-temporels des paysages steppiques d'Algérie : Cas de la région de Mechéria. Rev. Eco. 72 (1): 33-47, doi : 10.1016/j.scitotenv.2017.07.058

Idda S., Bonté B., Mansour H., Bellal S., Kuper M., 2017. Monument historique ou système bien vivant? Les foggarasdes oasis du Touat (Algérie) et leur réalimentation en eau par pompage. Cah. Agric. 26: 55007, doi: 10.1051/cagri/2017049

Kanoun M., Kanoun A., Baira M., Ziki B., 2007. Les produits de l'élevage ovins steppique : cas des agneaux de Djelfa. Options Méditerr. Sér. A (78): 211-217

Kanoun M., Huguenin J., Meguellati A., Ziki B., 2013. Capacité d'adaptation des exploitations d'élevages. Rencontres Rech. Rum., 20: 257-260

\section{Summary}

Gaci D., Huguenin J., Kanoun M., Boutonnet J.-P., Abdelkrim H. New pastoral movements: the case of sheep herders in Djelfa Wilaya, Algeria

The socioeconomic, demographic, political and climatic changes that the Algerian steppe has undergone in recent decades have led to major alterations in livestock husbandry practices, particularly in terms of pastoral mobility. This study describes new mobility practices currently adopted by herders in the region of Djelfa. It was based on 59 semidirective interviews conducted between 2014 and 2016 with herders in various hosting areas. Our results revealed a diversity of pastoral practices related to the distance traveled during an annual cycle, the calendar, the transhumance itinerary, and whether or not they passed through their home areas. The movements could vary from one year to another or during the herders' lifetime. This study showed that new forms of adaptation to the changing context of the Algerian steppe could be implemented by breeders through new long-distance movements. Our results highlight new forms of pastoral mobility despite the tightening of space and show that settling is not the only form of adaptation of pastoral populations to changes.

Keywords: sheep, transhumance, steppes, pastoral systems, Algeria
Kanoun M., 2016. Adaptation des éleveurs ovins face aux multiples changements d'ordre environnementaux et socioéconomique dans les territoires steppiques : cas des agropasteurs d'El Guedid. Thèse Doct. Ecole nationale supérieure d'agronomie, El Harrach, Algérie, 208 p.

Kanoun M., Bellahrache A., Meguellati-Kanoun A., Huguein J., Benedir M. 2018. Transhumance chez les agropasteurs ovins de Djelfa (Algerie) : quel type pour quel avenir? Alg. Jour. Arid. Envi., 8: 68-78

Kheffache H., 2018. Combinaison et hybridation des formes de coordination de la qualité : Cas de la filière lait de la wilaya de Médéa en Algérie. Thèse Doct., Supagro, Montpellier, France, Ecole nationale supérieure d'agronomie, El Harrach, Algérie. 442 p.

Kheldoun A., 2000. Evolution technologique et pastoralisme dans la steppe algérienne : le cas du camion Gak dans les hautes plaines steppiques occidentales. Option méditerr, Sér. A (39) : 221-127

Kherri A., 2013. Support pédagogique de cours $n^{\circ} 2$ Echantillonnage, www. cours-examens.org/images/An-2018/Etudes-superieures/Statistiques/6-2.pdf

Le Houérou H.N., 1995. Considération bioclimatologie et biogéographie des steppes arides du nord de I'Afrique. Options Méditerr., Sér. B (10) : 396 p.

Nedjraoui D., Bédrani S., 2008. La désertification dans les steppes algériennes : causes, impacts et actions de lutte. Vertigo 8. doi: 10.4000/vertigo. 5375

Nori M., 2017. Bergers étrangers, une opportunité pour le pastoralisme euro-méditerranéen?, Rev. Géo. Alpine., 105-4 : 16 p, doi : 10.4000/rga.3544

ONS, 2011. Recensement général de la population et de l'habitat, résultats 2008. Office national des statistiques, Alger, Algérie, 213 p.

Rondia P., 2006. Aperçu de l'élevage ovin en Afrique du nord. Rev. Filière Ovine Caprine 18: 11-14

ROSELT, 2005. Rapport final. Observatoire des hautes plaines steppiques du sud oranais, Biskra, Algérie, www.oss-online.org/sites/default/files/publications/oss-roselt-ni4-indicateurs_fr.pdf

Wenlin P., 2007. L'analyse de contenu comme méthode d'analyse qualitative d'entretiens : une comparaison entre les traitements manuels et l'utilisation de logiciels. Rech. Qual., 3: 243-272

Yabrir B., Laoun A., Chenouf N.S., Mati A., 2015. Caractéristiques des élevages ovins de la steppe centrale de l'Algérie en relation avec l'aridité du milieu : cas de la wilaya de Djelfa. Livest. Res. Rural Dev. 27: 207 (consulté 10 déc 2019)

\section{Resumen}

Gaci D., Huguenin J., Kanoun M., Boutonnet J.-P., Abdelkrim H. Nuevas posibilidades pastorales: caso de los criadores de ovinos del distrito (wilaya) de Djelfa, Argelia

Las evoluciones socioeconómica, demográfica, política y climática que a conocido la estepa argelina en el curso de las últimas décadas han inducido profundas transformaciones a nivel de las prácticas de crianza, particularmente en materia de movilidades pastorales. Este estudio describe nuevas prácticas de movilidad adoptadas actualmente por los criadores de la región de Djelfa. Se basó en 59 entrevistas semi directivas realizadas entre 2014 y 2016 con criadores de diferentes zonas de recepción. Nuestros resultados han evidenciado una diversidad de prácticas pastorales relativa a la distancia recorrida durante un ciclo anual, al calendario, al itinerario de trashumancia y al paso o no por el territorio de pertenencia. Estas movilidades pudieron variar de un año al otro o durante el curso de la vida de un criador. Este estudio mostró que las nuevas formas de adaptación al contexto cambiante de la estepa argelina podrían ser implementadas por los criadores mediante nuevas movilidades de gran distancia. Nuestros resultados realzan la renovación de las formas de movilidad pastoral a pesar del estrechamiento del espacio y muestran que la sedentarización no es la única forma de adaptación de las poblaciones pastorales a los cambios.

Palabras clave: ovino, trashumancia, estepas, sistemas pastorales, Argelia 
